\title{
Contribution of $d$-band electrons to ballistic transport and scattering during electron-phonon nonequilibrium in nanoscale Au films using an $a b$ initio density of states
}

\author{
Patrick E. Hopkins ${ }^{\text {a) }}$ \\ Engineering Sciences Center, Sandia National Laboratories, Albuquerque, New Mexico 87185-0346, USA \\ Derek A. Stewart \\ Cornell Nanoscale Science and Technology Facility, Cornell University, Ithaca, New York 14853-2700, USA
}

(Received 20 April 2009; accepted 23 July 2009; published online 8 September 2009)

\begin{abstract}
Electron-interface scattering during electron-phonon nonequilibrium in thin films creates another pathway for electron system energy loss as characteristic lengths of thin films continue to decrease. As power densities in nanodevices increase, excitations of electrons from sub-conduction-band energy levels will become more probable. These sub-conduction-band electronic excitations significantly affect the material's thermophysical properties. In this work, the role of $d$-band electronic excitations is considered in electron energy transfer processes in thin Au films. The electronic structure and density of states for gold are calculated using a plane wave pseudopotential density function approach. In thin films with thicknesses less than the electron mean free path, ballistic electron transport leads to electron-interface scattering. The ballistic component of electron transport is studied by a ballistic-diffusive approximation of the Boltzmann transport equation with input from $a b$ initio calculations. The effects of $d$-band excitations on electron-interface energy transfer are analyzed during electron-phonon nonequilibrium after short pulsed laser heating in thin films. () 2009 American Institute of Physics. [doi:10.1063/1.3211310]
\end{abstract}

\section{INTRODUCTION}

Routine production of nanomaterials has made devices with characteristic lengths on the order of nanometers readily available, as evident from the advent of the $45 \mathrm{~nm}$ transistor. As the sizes of these devices move toward atomistic limits, the applied power levels continue to increase to meet the research and development requirements. With this increase in power density in these devices, thermal management becomes the limiting engineering factor in future device design and implementation. ${ }^{1}$ At these nanoscale dimensions, the primary thermal resistance is due to interface scattering and is a key thermal management concern.

Interface scattering and thermal conductance across interfaces of nanomaterials have been the topics of several works over the past decades. ${ }^{1,2}$ Various forms of electron and phonon scattering processes have been studied to understand steady state heat dissipation. However, high power densities are possible in nanoscale devices, and this can give rise to a new form of interfacial resistance based on nonequilibrium electron-phonon scattering. As dimensions decrease and power levels increase, the electric field strength imposed on electrons in these devices drastically increases. The electric field, being directly related to the force imparted on the electron system, generates very energetic electrons that are far from equilibrium with the crystalline lattice. The energy density of the nonequilibrium electron system can be characterized by an electron temperature, which can be several thousands of Kelvin above the lattice temperature, depending on

\footnotetext{
${ }^{a)}$ Author to whom correspondence should be addressed. Electronic mail: pehopki@sandia.gov.
}

the source of the excitation. This nonequilibrium electronphonon state adds an increased complexity to interfacial scattering, and an additional thermal resistance as power levels increase and characteristic sizes decrease.

Electron-phonon nonequilibrium distributions have been studied since some of the earliest theories by Kaganov et al. ${ }^{3}$ and Anisimov et $a l^{4}$ The importance of understanding the thermal processes associated with electron-phonon rethermalization has proven critical in applications such as ablation and laser machining of materials, ${ }^{5,6}$ spin dynamics in magnetic devices, ${ }^{5-11}$ and thermal processes in metal film interconnects. ${ }^{12-14}$ Recent studies on electron-phonon energy transfer in nanostructures have found that there exists a change in the rate of electron-phonon energy transfer in the presence of an interface. ${ }^{15-19}$ Hopkins et al. ${ }^{16}$ attributed this change in electron-phonon equilibration to increased electron scattering at the interface between the nanomaterial and the substrate/host due to ballistic transport of the electrons after absorption of an ultrashort laser pulse. Ballistic electroninterface scattering was later investigated by Norris and Hopkins $^{20}$ with a ballistic-diffusive treatment to the Boltzmann transport equation (BTE), which predicted similar values and trends to the observed rates of electron-interface scattering.

The previous studies focused on low energy perturbation regimes, where electron systems in the nanometals can be treated by the free electron model. ${ }^{21}$ However, the electronic properties of the metal nanomaterials can deviate drastically from the free electron model. For example, the $d$-band edge in $\mathrm{Au}$ lies $\sim 2.4 \mathrm{eV}$ below the Fermi level. ${ }^{22}$ This causes deviation from the free electron model prediction of the thermophysical properties of $\mathrm{Au}$ at electron temperatures as low 
as $\sim 3500 \mathrm{~K}^{23}$ This $d$-band excitation effect and deviation from free electron predictions is observed in several other metals, at typically lower temperatures than $\mathrm{Au}$, due to the relative location of the $d$-bands in relation to the materials' Fermi energy. ${ }^{24}$ This magnitude of electron temperature change corresponds to an absorbed laser fluence of $\sim 10 \mathrm{~J} \mathrm{~m}^{-2}$, which is a reasonable fluence in applications related to laser processing and machining of nanomaterials. This electron temperature increase is also realized in $100 \mathrm{~nm}$ thick transistors upon gate voltages on the order of $-1.0 \mathrm{~V}^{25}$

In this paper, the effects of $d$-band to Fermi level electron excitations on ballistic electron transport and electroninterface scattering in thin $\mathrm{Au}$ films are studied using the ballistic-diffusive approximation (BDA) to the BTE. ${ }^{26,27}$ In Sec. II, calculations of pertinent thermophysical properties of $\mathrm{Au}$ are presented using both an $a b$ initio density of states (DOS), which includes the $5 d^{10}$ bands of $\mathrm{Au}$, and an approximate parabolic DOS assuming only the conduction band. In Sec. III, the ballistic and diffusive equations of the electron BTE for thin films are discussed and electron-interface scattering is studied in the free electron limit; that is, assuming only conduction band electrons are participating in transport. In Sec. IV, the $d$-band electrons' effects on electron processes are studied using the BTE calculations from Sec. III with the $a b$ initio DOS presented in Sec. II, which include $d$-band effects. The error when not considering $d$-band excitations in ballistic and diffusive transport predictions is discussed.

\section{DOS CALCULATIONS AND THERMOPHYSICAL PROPERTIES OF AU}

In the free electron limit, thermophysical properties of metal are typically calculated with a Sommerfeld expansion around the Fermi energy. ${ }^{28}$ Approximating the spectral DOS per unit volume of the free electrons in the conduction band as

$$
D(\varepsilon)=\frac{3 N_{\text {con }}}{2} \frac{1}{\varepsilon_{F}} \sqrt{\frac{\varepsilon}{\varepsilon_{F}}},
$$

where $N_{\text {con }}$ is the conduction band number density, $\varepsilon$ is the electron energy, and $\varepsilon_{F}$ is the Fermi energy assuming only the conduction band, gives rise to the following expression for the chemical potential as a function of temperature:

$$
\mu=\mu\left(T_{e}\right)=\varepsilon_{F}\left[1-\frac{1}{3}\left(\frac{\pi k_{B} T_{e}}{2 \varepsilon_{F}}\right)^{2}\right],
$$

where $k_{B}$ is the Boltzmann constant and $T_{e}$ is the electron temperature. The electron heat capacity ${ }^{28}$ and electronphonon coupling factor ${ }^{29}$ are given by

$$
C_{e}=C_{e}\left(T_{e}\right)=\int_{-\infty}^{\infty} \varepsilon D(\varepsilon) f_{0} d \varepsilon
$$

and

$$
G=G\left(T_{e}\right)=\pi \hbar k_{B} \lambda\left\langle\omega^{2}\right\rangle \int_{-\infty}^{\infty} \frac{[D(\varepsilon)]^{2}}{D\left(\varepsilon_{F}\right)}\left(-\frac{\partial f_{0}}{\partial \varepsilon}\right) d \varepsilon,
$$

where $\hbar$ is the reduced Planck's constant, $\lambda$ is the dimensionless electron-phonon mass enhancement parameter, ${ }^{30}\left\langle\omega^{2}\right\rangle$ is the second moment of the phonon spectrum, ${ }^{31} D\left(\varepsilon_{F}\right)$ is the DOS at the Fermi energy, and $f_{0}=f_{0}\left(\varepsilon, \mu, T_{e}\right)$ is the FermiDirac distribution function. Assuming that only the conduction band electrons contribute to heat storage and phonon energy loss, Eqs. (3) and (4) reduce to $C_{e}=\gamma T_{e}$ and $G=G_{0}$ $=\pi \hbar k_{B} \lambda\left\langle\omega^{2}\right\rangle D_{C}\left(\varepsilon_{F}\right)$, respectively, where $G_{0}$ is the original expression derived by Allen. ${ }^{32}$ Note that in noble metals, Eq. (4) reduces to $G_{0}$ even at high temperatures due to the large separation of the $d$-bands from the Fermi energy in these systems. ${ }^{33} \mathrm{In} \mathrm{Au}$, the Sommerfeld coefficient $\gamma$ is $62.9 \mathrm{~J} \mathrm{~m}^{-3} \mathrm{~K}^{-2}$ and $G_{0}=2.43 \times 10^{16} \mathrm{~W} \mathrm{~m}^{-3} \mathrm{~K}^{-1}$. The dashed lines in Fig. 1 show the calculations of Eqs. (1)-(4) for Au assuming $N_{\text {con }}=5.9 \times 10^{28} \mathrm{~m}^{-3}$ and $\varepsilon_{F}=5.53 \mathrm{eV}$ for electron temperatures up to $20000 \mathrm{~K}$.

Assuming that only the conduction band affects $\mu, C_{e}$, and $G_{0}$ is valid only at low temperatures when $d$-band electrons are not thermally excited. The temperature range of validity of the free electron (conduction band only) approximation is different for every metal as it depends on the location of the $d$-band with respect to the Fermi energy. ${ }^{24}$ In noble metals, this temperature range is larger than in other metals due to the large separation of the $d$-bands from the Fermi energy. To determine the effects of $d$-band excitations on $\mu, C_{e}$, and $G_{0}$, the DOS of the $d$-bands must be known.

The DOS of gold was calculated using a plane wave pseudopotential density functional approach $^{34}$ as implemented in the code QUANTUM ESPRESSO. ${ }^{35}$ The PerdewZunger form for the local density approximation (LDA) was assumed for exchange and correlation effects in the metal. ${ }^{36}$ Gold atoms were described using an ultrasoft pseudopotential. ${ }^{37}$ A plane wave cutoff of 70 Ry was used in all calculations, and a Monkhorst-Pack $k$-point mesh $^{38}$ of $48 \times 48 \times 48$ was used for integrations in the first Brillouin zone. The calculated equilibrium lattice constant and the bulk modulus were determined to be $4.047 \AA$ and 181.34 $\mathrm{GPa}$, respectively. The experimental lattice constant and bulk modulus for fcc $\mathrm{Au}$ are $4.0786 \AA$ and $172.8 \mathrm{GPa}$, respectively. ${ }^{39}$ The predicted values agree well with previous first principles studies of $\mathrm{Au}^{40}$ The tetrahedron approach was used to determine the DOS based on a fine $k$-grid in the Brillouin zone. ${ }^{41}$

The total DOS as a function of energy from the Fermi energy of the conduction bands and the $d$-bands in $\mathrm{Au}$ is shown by the solid lines in Fig. 1(a). The general features of the total DOS are very similar to that found in Ref. 24. However, there are subtle differences in the DOS due to how exchange and correlation are represented that will affect the calculated heat capacity and electron phonon coupling. In the current calculations, the LDA is used, which typically overbinds atoms in comparison to real systems. In Ref. 24, the generalized gradient approximation (GGA) was used, which typically underbinds and predicts lattice constants that are too large. They found a gold lattice constant of $4.175 \AA$, which is greater than the experimental value $(4.08 \AA)$. This difference in predicted lattice constants will also affect the calculated DOS. As atoms are brought together to form a solid, electronic bands form and continue to broaden. This results in the DOS based on LDA calculations (overbinding) having a slightly broader band and smaller DOS peaks than 

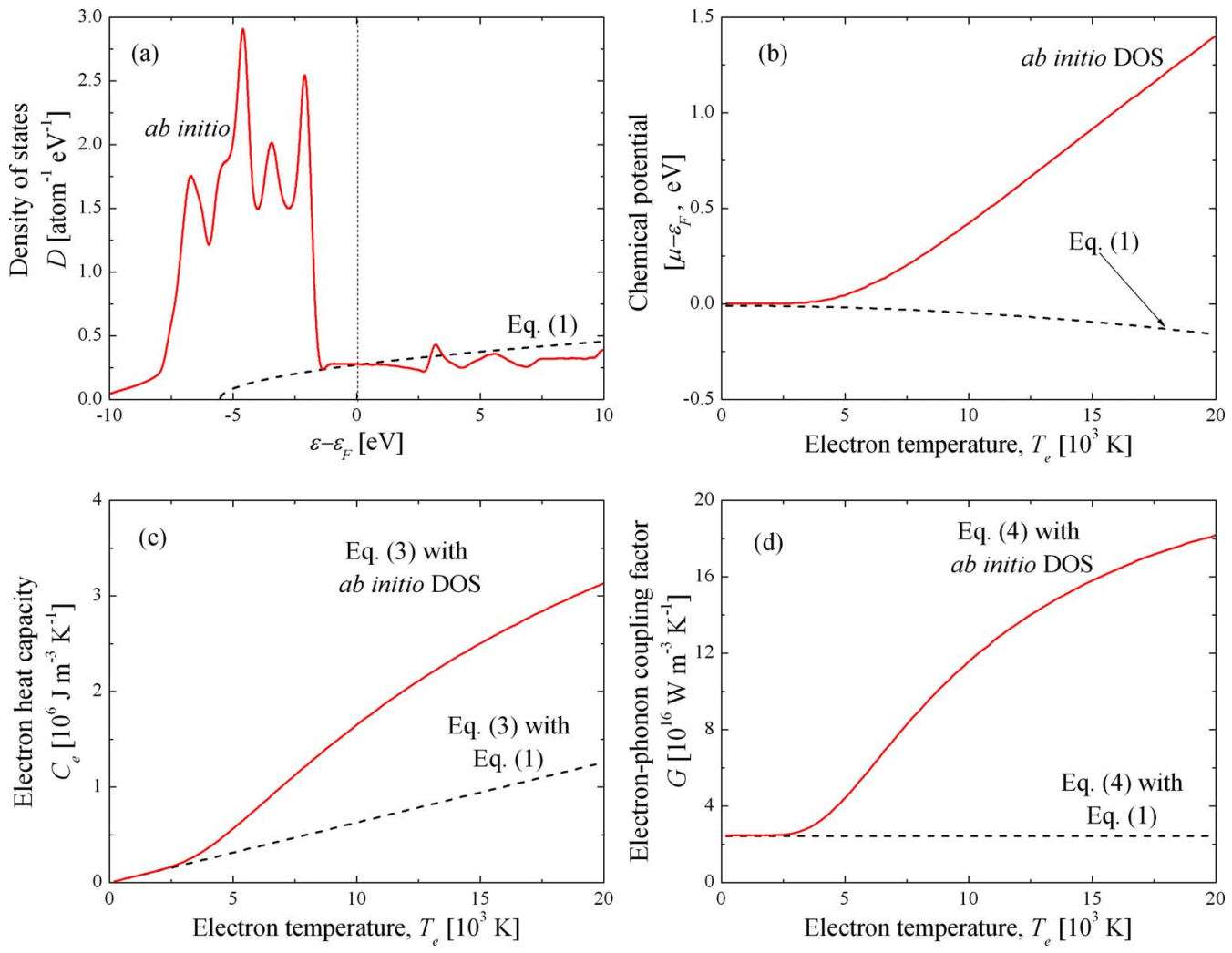

FIG. 1. (Color online) (a) Electron DOS, $D$; (b) chemical potential, $\mu$; (c) electron heat capacity, $C_{e}$; and (d) electron-phonon coupling factor, $G$, of Au using an approximate DOS that only takes into account a parabolic conduction band in Au described by Eq. (1) (dashed lines) and an $a b$ initio DOS that includes the $5 d^{10}$ bands (solid lines).

that found in the GGA calculation (underbinding). Comparison of Fig. 1(a) with Fig. 4 of Ref. 24 supports this analysis.

At high temperatures, Fermi smearing thermally excites electrons from the $d$-bands near the Fermi energy, drastically affecting calculations of $\mu, C_{e}$, and $G_{0}$, as shown by the solid lines in Figs. 1(b) $-1(\mathrm{~d})$, respectively. The chemical potential was calculated with the procedure outlined by Lin et al. ${ }^{24}$ The $C_{e}$ and $G_{0}$ data in Figs. 1(c) and 1(d) are calculated with Eqs. (3) and (4), respectively, using the ab initio DOS. These calculations are similar to those presented by Lin $e t$ al. ${ }^{24} \mathrm{In}$ $\mathrm{Au}$, significant deviations from the free electron approximation in $\mu, C_{e}$, and $G_{0}$ are apparent at $\sim 3500 \mathrm{~K}$. This represents the onset of thermal $d$-band excitations. The effects of these $d$-band excitations on ballistic and diffusive electron transport during electron-phonon nonequilibrium are discussed in the remainder of this work.

\section{BALLISTIC AND DIFFUSIVE ELECTRON TRANSPORTS IN THIN FILMS: FREE ELECTRON APPROXIMATION}

\section{A. Equation of electron energy transfer}

The BTE for electron transport in one dimension is given by ${ }^{42,43}$

$$
\frac{\partial f}{\partial t}+v_{z} \frac{\partial f}{\partial z}+\frac{F_{z}}{m} \frac{\partial f}{\partial v_{z}}=\left(\frac{\partial f}{\partial t}\right)_{c},
$$

where $f$ is the nonequilibrium electron probability distribution, $v_{z}$ is the electron velocity in the $z$-direction, $F_{z}$ is the Lorentz force in the $z$-direction, and the term $(\partial f / \partial t)_{c}$ is the time rate of change in the nonequilibrium distribution due to electron collisions. In this analysis, the $z$-direction is taken as perpendicular to an interface that provides resistance to heat flow-the cross plane direction. In the thin film limit-that is, when the film thickness is less than the thermal penetration depth-Eq. (5) reduces to

$$
\frac{\partial f}{\partial t}=\left(\frac{\partial f}{\partial t}\right)_{c},
$$

since the thin film is much smaller than the thermal wave emitted into the film, and therefore the spatial gradients in the film are negligible. The thermal penetration depth during electron-phonon nonequilibrium can be estimated by $\delta_{\text {th }}$ $\approx \sqrt{k / G}$, where $k$ is the electron system thermal conductivity. ${ }^{44}$ At room temperature, Au has a thermal conductivity of $317 \mathrm{~W} \mathrm{~m}^{-1} \mathrm{~K}^{-1}$ (Ref. 45) and an electronphonon coupling factor of $2.2 \mathrm{~W} \mathrm{~m}^{-3} \mathrm{~K}^{-1},{ }^{12,46}$ giving a thermal penetration depth of $\delta_{\mathrm{th}} \approx 120 \mathrm{~nm}$. With Eq. (6), the equation of electron energy transfer (EEET), ${ }^{20}$ which parallels the equation of phonon radiative transport, ${ }^{47}$ is given by

$$
\frac{\partial U_{\varepsilon}(t)}{\partial t}=\left(\frac{\partial U_{\varepsilon}(t)}{\partial t}\right)_{c},
$$

where $t$ is the time and $U_{\varepsilon}$ is the volumetric electron energy density per unit energy defined as

$$
U_{\varepsilon}=\varepsilon D(\varepsilon) f(1-f) \text {. }
$$

In this development, the factor of $(1-f)$ is included since the goal is to study energy transfer, which can only occur 
when there are empty states in the vicinity of occupied states. For example, the quantity $\int_{\varepsilon} \varepsilon D(\varepsilon) f d \varepsilon$ gives the total energy of the electron system. However, in electron energy transfer, only electron energies with empty states at or around that energy will participate in energy transfer processes. Therefore, the probability that a state is occupied and that there is an unoccupied state at that energy is given by $f(1-f)$, so that the electron energy density participating in energy transfer processes is estimated by Eq. (8).

Equation (7) is subject to

$$
U(t=0)=\frac{A}{d},
$$

where $A$ is the absorbed energy per unit area by the electron system and $d$ is the film thickness. yields

Applying a relaxation time approximation to the EEET

$$
\frac{\partial U_{\varepsilon}(t)}{\partial t}=-\frac{U_{\varepsilon}(t)-U_{\varepsilon, 0}\left(T_{e}\right)}{\tau},
$$

where $U_{\varepsilon, 0}\left(T_{e}\right)=\varepsilon D(\varepsilon) f_{0}\left(1-f_{0}\right)$ is the equilibrium electron energy density available for transport. In Eq. (6), $\tau$ is the relaxation time of the electrons in the metal films, which for purely diffusive transport is the electron-phonon thermalization time, $\tau_{e p}$.

\section{B. BDA to the EEET: No substrate energy loss}

Applying the BDA to electron transport requires a slightly different formulation than the BDA for phonon transport, specifically the single relaxation time approximation. Upon energy absorption, the electron system temperature evolution and energy transfer can be divided into two characteristic time intervals. The first time interval is the electron-electron relaxation time, $\tau_{e e}$, which represents the time it takes for the electron system to relax into an equilibrium Fermi-Dirac distribution. Ballistic transport of electrons occurs during this time. Once equilibrium is achieved in the electron system, the high temperature electrons transmit energy to the lattice through electron-phonon scattering processes over $\tau_{e p}$. In metals, typical electron-electron relaxation times are from tens to hundreds of femtoseconds and typical electron-phonon thermalization times are a few picoseconds. ${ }^{12,48}$ Due to the different characteristic times governing ballistic and diffusive (electron-phonon) transports, ballistic electron transport must be modeled with $\tau_{e e}$ and diffusive electron transport must be modeled with $\tau_{e p}$.

Following the ballistic-diffusive approximation to the BTE, ${ }^{26,27}$ the ballistic and diffusive electron energy densities can be separated and the EEET can be rewritten as

$$
\begin{aligned}
\frac{\partial U_{\varepsilon}}{\partial t} & =\frac{\partial}{\partial t}\left[U_{\varepsilon, b}(t)+U_{\varepsilon, m}(t)\right] \\
& =-\frac{U_{\varepsilon, b}-U_{\varepsilon, m}}{\tau_{e e}}-\frac{U_{\varepsilon, m}-U_{\varepsilon, 0}}{\tau_{e p}},
\end{aligned}
$$

where the subscript $b$ refers to the ballistic component and the subscript $m$ refers to the diffusive component (generated in the medium). Equation (11) assumes that the ballistic component relaxes to the diffusive component, which in turn relaxes to the equilibrium distribution. In the electron scattering and energy thermalization processes discussed above, during electron-electron scattering processes and Fermi relaxation, the nonequilibrium electron distribution of the ballistic electrons relaxes to a Fermi distribution, so $U_{\varepsilon, m}(0)$ $=U_{\varepsilon, 0}\left(T_{e}\right)$. During electron-phonon scattering processes, $U_{\varepsilon, 0}\left(T_{e}\right)$ then relaxes to $U_{\varepsilon, 0}\left(T_{p}\right)$, where $T_{p}$ is temperature of the thermalized phonon system. The development of the BDA to the EEET assumes that electron-interface scattering is completely elastic; that is, during electron-electron scattering and Fermi relaxation, electron-interface scattering does not transfer energy away from the film electron system.

Relating the ballistic and diffusive terms, and recognizing that the incident laser energy is absorbed by the ballistic electron system, the BDA of the EEET for an electron system in which $\delta_{\text {th }} \leq d$ and no electron energy loss to the substrate during Fermi relaxation is given by

$$
\begin{aligned}
& \frac{\partial U_{\varepsilon, b}}{\partial t}=-\frac{U_{\varepsilon, b}-U_{\varepsilon, 0}\left(T_{e}\right)}{\tau_{e e}}, \\
& \frac{\partial U_{\varepsilon, m}}{\partial t}=-\frac{U_{\varepsilon, 0}\left(T_{e}\right)-U_{\varepsilon, 0}\left(T_{p}\right)}{\tau_{e p}},
\end{aligned}
$$

subject to

$$
U_{b}(0)=\frac{A}{d}
$$

and

$$
U_{m}(0)=U_{b}\left(\tau_{e e}\right) .
$$

The solutions of Eqs. (12)-(15) yield $U_{\varepsilon, b}(t)$ and $U_{\varepsilon, m}(t)$, given by

$$
U_{\varepsilon, b}(t)=U_{\varepsilon, 0}\left(T_{e}\right)\left(1-\exp \left[-\frac{t}{\tau_{e e}}\right]\right)+U_{\varepsilon, b}(0) \exp \left[-\frac{t}{\tau_{e e}}\right]
$$

and

$$
\begin{aligned}
U_{\varepsilon, m}(t)= & -\frac{U_{\varepsilon, 0}\left(T_{e}\right)-U_{\varepsilon, 0}\left(T_{p}\right)}{\tau_{e p}} t+U_{\varepsilon, 0}\left(T_{e}\right)\left(1-\frac{1}{e}\right) \\
& +\frac{U_{\varepsilon, b}(0)}{e},
\end{aligned}
$$

which are related to the average power transferred from the ballistic and diffusive electron systems via

$$
P_{b, \mathrm{av}}=\frac{1}{\tau_{e e}^{2}} \int_{0}^{\tau_{e e}} \int_{-\infty}^{\infty} U_{\varepsilon, b}(t) d \varepsilon d t
$$

and

$$
P_{m, \mathrm{av}}=\frac{1}{\tau_{e p}^{2}} \int_{0}^{\tau_{e p}} \int_{-\infty e}^{\infty} U_{\varepsilon, m}(t) d \varepsilon d t .
$$

Equation (18) represents the volumetric power transferred from the ballistic electron system. Since no substrate energy loss is assumed in this calculation, this represents the power density transferred from the ballistic electron system to the 
diffusive electron system. Equation (19) represents the volumetric power transferred from the diffusive electron system to the equilibrium system described by the Fermi distribution.

Note that this BDA gives the EEET in a per energy basis, but the source of the ballistic component [Eq. (14)] gives the total energy absorbed by all the electrons. This formulation could be extended to account for only specific electron energies, say, from photons inducing selected interband transitions. Since the internal energy of the electron system drastically changes when $d$-band electrons are thermally excited, to relate the amount of energy absorbed by the ballistic electron system to the energy absorbed by the electrons at a given energy, the number of electrons per unit energy must be known-i.e., the electron DOS. The energy absorbed by the ballistic electron system per electron energy is given by

$$
U_{\varepsilon, b}(0)=\frac{A}{d} \frac{D(\varepsilon) f_{0}\left(1-f_{0}\right)}{\int_{-\infty}^{\infty} D(\varepsilon) f_{0}\left(1-f_{0}\right) d \varepsilon}+U_{\varepsilon, b}\left(T_{e}\right),
$$

where $f_{0}$ is evaluated at $T_{e}$ so that Eq. (20) calculates the nonequilibrium energy density participating in conduction that is slightly perturbed from the energy associated with an electron system at temperature $T_{e}$. Note that the development of energy absorption by the electron system only considers thermal energies participating in conduction due to Fermi smearing and does not consider interband transitions causing a nonthermal repopulation of the electron energy bands. ${ }^{49}$ Also, for the same absorbed power, as temperature increases, the power is distributed to more electrons.

To solve for the ballistic and diffusive power densities transferred over the characteristic electron scattering times (electron-electron and electron-phonon) as a function of temperature, the electron DOS must be known. In these calculations, only the conduction band will be considered for electron energy transfer calculations [Eq. (1)]. This free electron approximation is only valid for low electron temperatures, ${ }^{23,24}$ as seen in Fig. 1, but will be considered here for temperatures up to $20000 \mathrm{~K}$, well above the limits of validity of the free electron approximation for Au. The $d$-bands will be considered later and the errors associated with the free electron approximation at these temperatures will be apparent.

The electron-phonon relaxation time, $\tau_{e p}$, can be estimated by

$$
\tau_{e p}=\left(\frac{C_{e} C_{p}}{C_{e}+C_{p}}\right) \frac{1}{G},
$$

where $C_{p}$ is the heat capacity of the phonon system, which for $\mathrm{Au}$ is $2.47 \times 10^{6} \mathrm{~J} \mathrm{~m}^{-3} \mathrm{~K}^{-1}$ at $300 \mathrm{~K}$, and $G$ is a constant at all electron temperatures when only considering conduction band energy transfer. ${ }^{33}$ Equation (21) is a relative electron-phonon thermalization time; that is, it represents the time for a Fermi-relaxed electron system at temperature $T_{e}$ to thermalize with a phonon system at temperature $T_{p}$. In this work, a constant phonon temperature of $300 \mathrm{~K}$ is assumed, which is valid during electron-phonon nonequilibrium applications since energy is not deposited into the phonon system until after a substantial number of electron-phonon scattering events (until near the electron-phonon thermalization time).

Figure 2(a) shows the ballistic volumetric power transfer as a function of electron temperature for four different electron-electron thermalization times, $\tau_{e e}=50,200,350$, and $500 \mathrm{fs}$, assuming an absorbed energy density of $1 \mathrm{~J} \mathrm{~m}^{-3}$. This effectively makes the source term, Eq. (9), negligible (small perturbation) and allows us to study only electron scattering and cooling processes, which is of interest in this work, without having to consider any heating or absorption processes. These calculations assume elastic-interface scattering (no energy loss to the substrate). These relaxation times were chosen since $\tau_{e e}$ in $\mathrm{Au}$ has been theoretically calculated to be as small as 50 fs (Ref. 48) and experimentally measured to be as large as $500 \mathrm{fs}{ }^{50}$ Figure 2(b) shows the ratio of ballistic to diffusive volumetric power transfer as a function of electron temperature. The ballistic component is one to two orders of magnitude larger than the diffusive component. Note that for all electron-electron thermalization times, the diffusive components are identical since the electron-phonon thermalization time is governed by Eq. (21). Although the trends are the same, the values reported here are different than those reported by previously for the BDA to the EEET (Ref. 51) in the thin film limit since in the present work a thermalized electron system is assumed as the starting point of diffusive transport.

\section{BDA to the EEET: Substrate energy loss}

In the case of elastic electron-interface scattering, the ballistic and diffusive components to electron transport are separated by considering two different EEETs: one for the electron-electron relaxation and one for the electron-phonon relaxation. However, in the case of inelastic electroninterface scattering, the ballistic carriers that inelastically scatter at the film/substrate interface experience no internal scattering in the film. In this case, the BDA of the EEET takes a different form than in the previous section.

To consider inelastic electron-interface scattering and electron system energy loss during Fermi relaxation, the form of the BDA to the EEET takes a form similar to Hopkins and Norris ${ }^{51}$ given by

$$
\frac{\partial U_{\varepsilon}}{\partial t}=\frac{\partial}{\partial t}\left(U_{\varepsilon, b i}+U_{\varepsilon, m}\right)=-\frac{U_{\varepsilon, b i}}{\tau_{e i}}-\frac{U_{\varepsilon, 0}\left(T_{e}\right)-U_{\varepsilon, 0}\left(T_{p}\right)}{\tau_{e p}},
$$

where the subscript $b i$ refers to the ballistic component inelastically scattering at the interface and $\tau_{e i}$ is the electroninterface relaxation time. Relating the ballistic and diffusive terms yields the BDA to the EEET assuming electron energy loss to the substrate during Fermi relaxation, given by

$$
\begin{aligned}
& \frac{\partial U_{\varepsilon, b i}}{\partial t}=-\frac{U_{\varepsilon, b i}}{\tau_{e i}}, \\
& \frac{\partial U_{\varepsilon, m}}{\partial t}=-\frac{U_{\varepsilon, 0}\left(T_{e}\right)-U_{\varepsilon, 0}\left(T_{p}\right)}{\tau_{e p}},
\end{aligned}
$$

subject to 

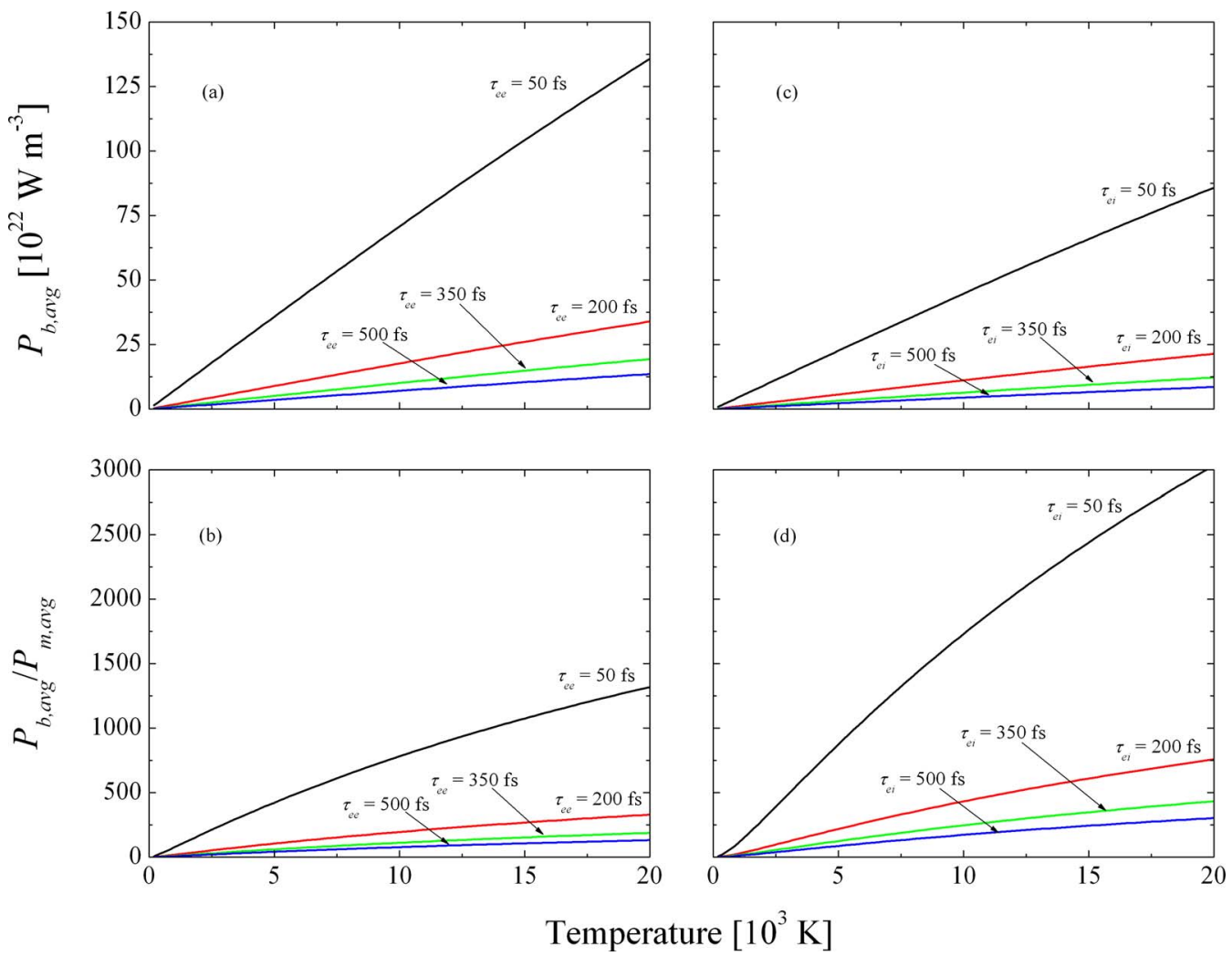

FIG. 2. (Color online) Assuming no energy loss to the substrate: (a) ballistic volumetric power transfer as a function of electron temperature for four different electron-electron thermalization times, $\tau_{e e}=50,200,350$, and $500 \mathrm{fs}$, assuming an absorbed energy density of $1 \mathrm{~J} \mathrm{~m}^{-3}$ in a $20 \mathrm{~nm} \mathrm{Au}$ film, and (b) ratio of ballistic to diffusive volumetric power transfer as a function of electron temperature. Assuming energy loss to the substrate: (c) ballistic volumetric power transfer as a function of electron temperature for four different electron-interface thermalization times, $\tau_{e i}=50,200,350$, and 500 fs, assuming an absorbed energy density of $1 \mathrm{~J} \mathrm{~m}^{-3}$ in a $20 \mathrm{~nm}$ Au film, and (d) ratio of ballistic to diffusive volumetric power transfer as a function of electron temperature. The increase in $P_{b, \text { av }}$ with temperature is related to the increase in volumetric internal energy, which increases with temperature and is greater than the decay of this internal energy over time.

$$
U_{\varepsilon, b i}(0)=\frac{A}{d} \frac{D(\varepsilon) f_{0}\left(1-f_{0}\right)}{\int_{-\infty}^{\infty} D(\varepsilon) f_{0}\left(1-f_{0}\right) d \varepsilon}+U_{\varepsilon, b}\left(T_{0}\right)
$$

and

$$
U_{\varepsilon, m}(0)=U_{\varepsilon, b i}(0)-U_{\varepsilon, b i}\left(\tau_{e i}\right) .
$$

The solutions to Eqs. (23)-(26) are given by

$$
U_{\varepsilon, b i}(t)=U_{\varepsilon, b i}(0) \exp \left[\frac{-t}{\tau_{e i}}\right]
$$

and

$$
U_{\varepsilon, m}(t)=-\frac{U_{\varepsilon, 0}\left(T_{e}\right)-U_{\varepsilon, 0}\left(T_{p}\right)}{\tau_{e p}} t+U_{\varepsilon, b i}(0)-U_{\varepsilon, b i}\left(\tau_{e i}\right) .
$$

The average power transferred from the ballistic and diffusive electron systems assuming inelastic electron-interface scattering during Fermi relaxation of the electron system are given by Eqs. (18) and (19) with Eqs. (27) and (28), and are denoted $P_{b i \text {,av }}$ and $P_{m i \text {,av }}$, respectively. Here, the ballistic power transfer represents the volumetric power transferred from the ballistic electron system to the equilibrium substrate system. Note that the initial condition of the diffusive equation assumes that the energy of the electron system that experiences inelastic-interface scattering and substrate coupling is lost from the film system. Figure 2(c) shows the ballistic volumetric power transfer as a function of electron temperature for four different electron-interface thermalization times, $\tau_{e i}=50,200,350$, and $500 \mathrm{fs}$, assuming an absorbed energy density of $1 \mathrm{~J} \mathrm{~m}^{-3}$. These calculations assume inelasticinterface scattering, that is, upon interface scattering, electrons lose energy from the film system to the substrate. In this case, the energy lost from the film electron system over the electron-interface thermalization time [see Fig. 2(c)] is not available to couple with the film phonon system. Note the trends are the same as in Fig. 2(a), but the values are different for the same input power, since energy is lost to the substrate system. This causes the ballistic component to decrease; note that the ballistic component represents the energy lost to the substrate during electron-electron relaxation. However, the ratio of the ballistic component to diffusive component increases [see Fig. 2(d)] (ratio of power lost to substrate to power lost to phonon system) since the diffusive component does not decrease as much as the difference in power transfer predicted between Figs. 2(a) and 2(c).

As previously mentioned the power calculations thus far have assumed no $d$-band influence. The error associated with this assumption will be apparent at high temperatures as discussed later. However, these calculations are validated at low temperatures $\left(T_{e}<3500 \mathrm{~K}\right)$ by comparing the results to the data from Hopkins et al. ${ }^{16}$ Hopkins et al. ${ }^{16}$ measured the electron-phonon coupling factor of Au films in the thin film 


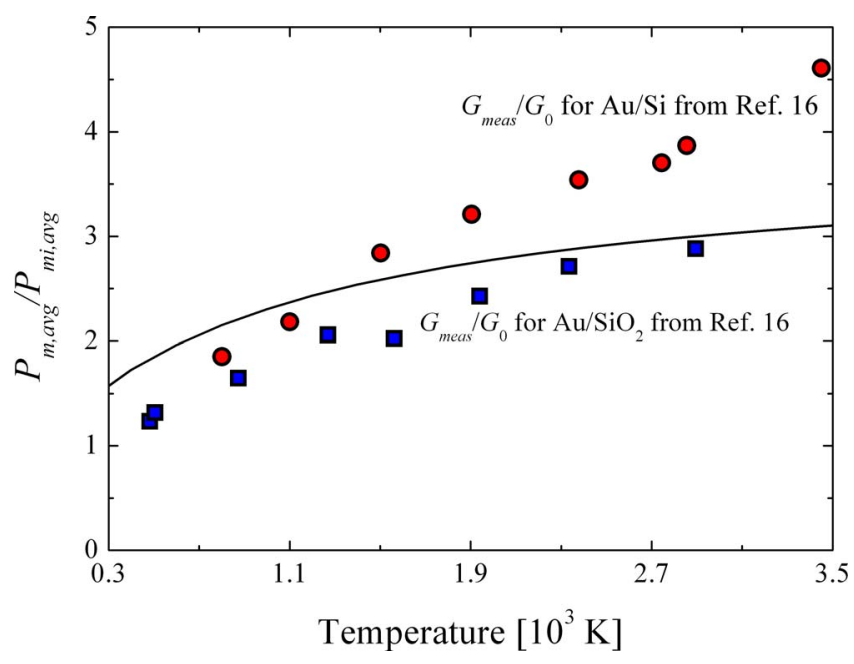

FIG. 3. (Color online) Ratio of elastic electron-interface scattering to inelastic electron-interface scattering diffusive power densities, $P_{m \text {,av }} / P_{m i \text { av }}$ compared to the measured electron-phonon coupling factor in thin Au films by Hopkins et al. (Ref. 16) normalized by $G_{0}$.

(homogeneous heating assuming no temperature gradient) limit after electron-electron thermalization with a transient thermoreflectance technique. By analyzing the data with a two-temperature model ${ }^{4}$ that assumes a completely insulated film/substrate boundary (i.e., elastic electron-interface scattering), they measured $G$ in the Au films to be greater than $G_{0}$ at low temperatures, where theory [Fig. 1(d)] predicts a constant $G$. Therefore, their measured $G, G_{\text {meas }}$, represents the power transfer from the electron to phonon system assuming no substrate energy loss since their data were analyzed assuming an insulated boundary condition. Dividing $G_{\text {meas }}$ by $G_{0}$ gives an estimate of the ratio of electron-phonon (diffusive) power transfer assuming elastic electron-interface scattering to electron-phonon power transfer assuming inelastic scattering (note that using a model that takes into account inelastic electron-interface scattering, such as the three-temperature model, ${ }^{52}$ should result in a measured $G$ equal to $G_{0}$ at low temperatures). Therefore, comparing $G_{\text {meas }} / G_{0}$ from Hopkins et al. ${ }^{16}$ to $P_{m, \text { av }} / P_{m i \text {,av }}$ gives a benchmark for these calculations and the formulation of the BDA for the EEET in the thin film limit. These results are shown in Fig. 3 and show good agreement with no fitting parameters required. The difference in trends between the data and the model at higher temperatures could be due to various interfacial scattering mechanisms that are discussed by Hopkins et al. ${ }^{16}$ but not explicitly taken into account in this development.

\section{BALLISTIC AND DIFFUSIVE ELECTRON TRANSPORTS IN THIN FILMS: D-BAND ELECTRONS}

The BDA to the EEET in the free electron limit for two cases is given as follows: (1) Elastic electron-interface scattering-i.e., no energy loss into the underlying substrate as a result of electron-interface scattering; and (2) inelastic electron-interface scattering-i.e., energy loss into the underlying substrate as a result of electron-interface scattering. Assuming the free electron limit, only the parabolic conduction band was considered in the analysis and calculations were performed for electron temperatures up to $20000 \mathrm{~K}$. However, in $\mathrm{Au}$, at temperatures greater than $\sim 3500 \mathrm{~K}$, thermal $d$-band excitations can drastically affect the thermal properties, and, therefore, the power transfer will be affected.

To determine the effects of $d$-band excitations on the power transfer calculations, the DOS of the $d$-band must be taken into account. Using the $a b$ initio DOS along with the thermophysical properties in Fig. 1, the calculations in Fig. 2 are repeated in Fig. 4. These are the same calculations as Fig. 2 only taking into account the $5 d^{10}$ bands in Au by using the $a b$ initio DOS to account for the influence of $d$-band excitation on ballistic and diffusive power transfers in thin $\mathrm{Au}$ films. In addition, Eqs. (3) and (4) are used with Eq. (21) to evaluate $\tau_{e p}$ and correctly account for the temperature trends due to $d$-band excitations. Figure 4 shows the calculations in Fig. 2 with the inclusion of the $d$-bands. The predictions show drastically different trends and values than those in Fig. 2 , which do not take into account the $d$-bands. The onset of the $d$-band participation is clear at about $3500 \mathrm{~K}$. Note that for temperatures less than the onset of $d$-band influence, the predictions of Figs. 2 and 4 are nearly identical, as expected from the agreement between the various thermophysical properties shown in Fig. 1 with the two calculation methods shown in Fig. 1. The $d$-band excitations cause the ballistic power transfer to increase and exhibit a nonlinear trend. The ratio of the ballistic to diffusive components shows an inflection point around $5000 \mathrm{~K}$, indicating that as temperature increases past the initial onset of $d$-band excitations, the diffusive component increases when considering the $d$-bands more so than when considering only the conduction band. This is intuitive when examining the trends in $G$ with and without $d$-band inclusion, as shown in Fig. 1(d). The inclusion of $d$-bands causes a drastic increase in the ballistic power transfer as compared to the case of no $d$-band excitations, which in turn affects the diffusive power transfer and ratio of ballistic to diffusive power transfer. Assuming inelastic electron-interface scattering, the electrons lose significantly more energy to the substrate when considering the underlying $d$-band excitations. Also, the ratio of ballistic to diffusive power transfer increases from the elastic-interface scattering cases, but exhibit the same trends. This is a similar result to the calculations in Fig. 2.

\section{CONCLUSIONS}

In high powered nanosystems, electron-interface scattering can create significant thermal resistances during electronphonon nonequilibrium heating. As characteristic sizes decrease, ballistic electron transport could cause electron system energy loss from electron-interface scattering that can alter the thermal resistive processes in a material. In this work, the ballistic-diffusive approximation to the BTE is applied to the EEET to study the effects of electron-interface scattering on electron power transfer processes in thin films. At high electron temperatures, $d$-band excitations can change the thermal properties around the Fermi energy, drastically altering the predicted power transfer during ballistic and diffusive electron transport processes. In high temperature 

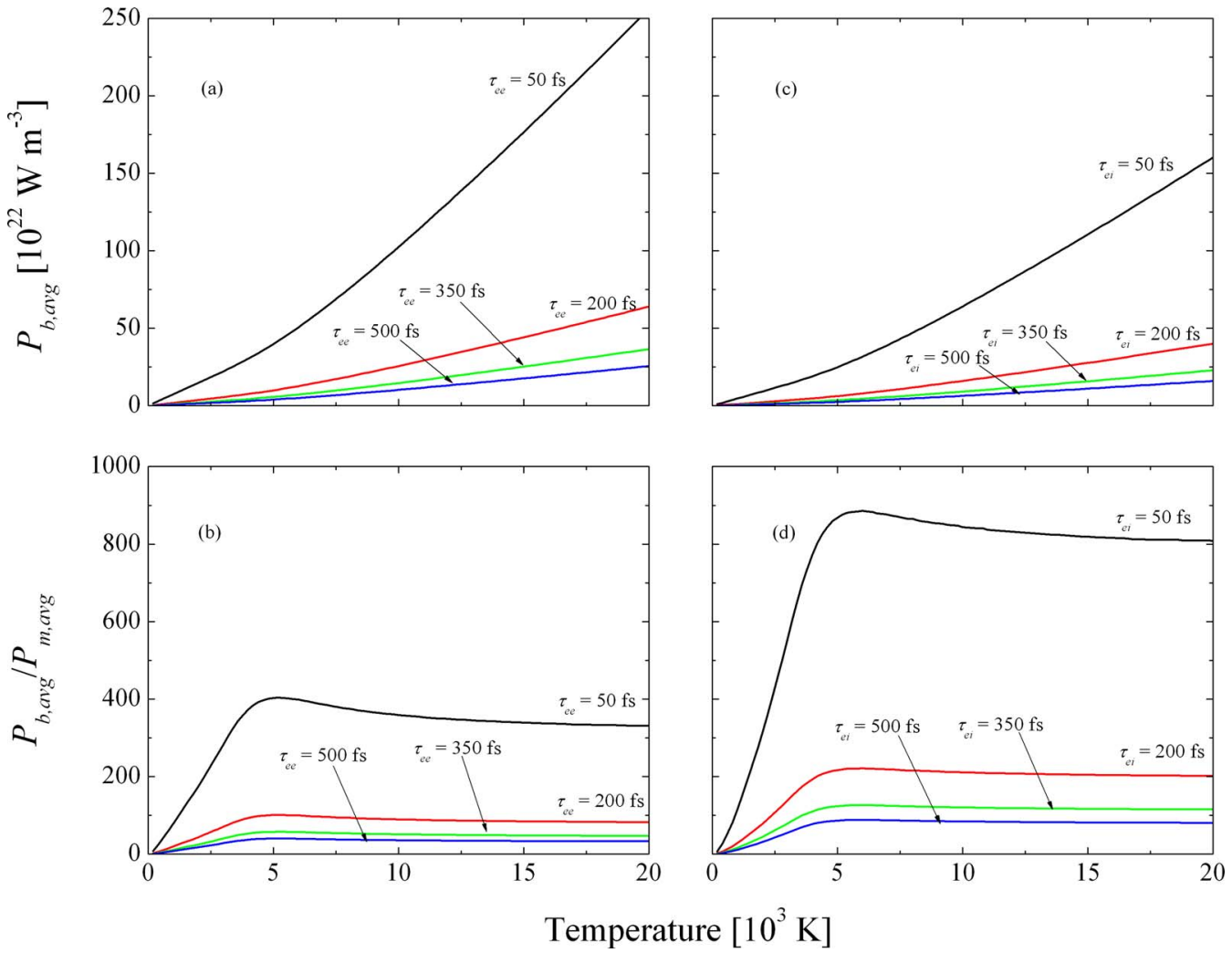

FIG. 4. (Color online) Same calculations as Fig. 2 only taking into account the $5 d^{10}$ bands in Au by using the ab initio DOS of $d$-band excitation on ballistic and diffusive power transfers in thin Au films.

electron-phonon nonequilibrium transport studies, the $d$-band must be considered for accurate predictions of electron energy transport.

\section{ACKNOWLEDGMENTS}

P.E.H. is greatly appreciative for funding from the LDRD program office through the Sandia National Laboratories Harry S. Truman Fellowship. Sandia is a multiprogram laboratory operated by Sandia Corporation, a LockheedMartin Co., for the U.S. Department of Energy's National Nuclear Security Administration under Contract No. DEAC04-94AL85000. First principles calculations for Au were performed on the Intel Cluster at the Cornell Nanoscale Facility, which is part of the National Nanotechnology Infrastructure Network funded by the National Science Foundation.

${ }^{1}$ D. G. Cahill, W. K. Ford, K. E. Goodson, G. D. Mahan, A. Majumdar, H. J. Maris, R. Merlin, and S. R. Phillpot, J. Appl. Phys. 93, 793 (2003).

${ }^{2}$ E. T. Swartz and R. O. Pohl, Rev. Mod. Phys. 61, 605 (1989).

${ }^{3}$ M. I. Kaganov, I. M. Lifshitz, and L. V. Tanatarov, Sov. Phys. JETP 4, 173 (1957).

${ }^{4}$ S. I. Anisimov, B. L. Kapeliovich, and T. L. Perel'man, Sov. Phys. JETP 39, 375 (1974).

${ }^{5}$ D. S. Ivanov and L. V. Zhigilei, Phys. Rev. B 68, 064114 (2003).

${ }^{6}$ S.-S. Wellershoff, J. Hohlfeld, J. Gudde, and E. Matthias, Appl. Phys. A: Mater. Sci. Process. 69, S99 (1999).

${ }^{7}$ E. Beaurepaire, J.-C. Merle, A. Daunois, and J.-Y. Bigot, Phys. Rev. Lett. 76, 4250 (1996).

${ }^{8}$ L. Guidoni, E. Beaurepaire, and J.-Y. Bigot, Phys. Rev. Lett. 89, 017401 (2002).

${ }^{9}$ J. Hohlfeld, E. Matthias, R. Knorren, and K. H. Bennemann, Phys. Rev. Lett. 78, 4861 (1997).
${ }^{10}$ B. Koopmans, M. van Kampen, J. T. Kohlhepp, and W. J. M. de Jonge, J. Appl. Phys. 87, 5070 (2000).

${ }^{11}$ M. van Kampen, J. T. Kohlhepp, W. J. M. de Jonge, B. Koopmans, and R. Coehoorn, J. Phys.: Condens. Matter 17, 6823 (2005).

${ }^{12}$ J. Hohlfeld, S. S. Wellershoff, J. Gudde, U. Conrad, V. Jahnke, and E. Matthias, Chem. Phys. 251, 237 (2000).

${ }^{13}$ P. E. Hopkins, J. M. Klopf, and P. M. Norris, Appl. Opt. 46, 2076 (2007).

${ }^{14}$ J. L. Hostetler, A. N. Smith, D. M. Czajkowsky, and P. M. Norris, Appl. Opt. 38, 3614 (1999).

${ }^{15}$ C. A. C. Bosco, A. Azevedo, and L. H. Acioli, Appl. Phys. Lett. 83, 1767 (2003).

${ }^{16}$ P. E. Hopkins, J. L. Kassebaum, and P. M. Norris, J. Appl. Phys. 105, 023710 (2009).

${ }^{17}$ A. Arbouet, C. Voisin, D. Christofilos, P. Langot, N. Del Fatti, F. Vallee, J. Lerme, G. Celep, E. Cottancin, M. Gaudry, M. Pellarin, M. Broyer, M. Maillard, M. P. Pileni, and M. Treguer, Phys. Rev. Lett. 90, 177401 (2003).

${ }^{18}$ G. V. Hartland, Int. J. Nanotechnol. 1, 307 (2004).

${ }^{19}$ J. H. Hodak, A. Henglein, and G. V. Hartland, J. Chem. Phys. 112, 5942 (2000).

${ }^{20}$ P. M. Norris and P. E. Hopkins, ASME J. Heat Transfer 131, 043207 (2009).

${ }^{21}$ C. Kittel, Introduction to Solid State Physics, 7th ed. (Wiley, New York, 1996).

${ }^{22}$ N. E. Christensen and B. O. Seraphin, Phys. Rev. B 4, 3321 (1971).

${ }^{23}$ Z. Lin and L. V. Zhigilei, Proc. SPIE 6261, 62610U (2006).

${ }^{24}$ Z. Lin, L. V. Zhigilei, and V. Celli, Phys. Rev. B 77, 075133 (2008).

${ }^{25}$ A. Majumdar, K. Fushinobu, and K. Hijikata, J. Appl. Phys. 77, 6686 (1995).

${ }^{26}$ G. Chen, Phys. Rev. Lett. 86, 2297 (2001).

${ }^{27}$ G. Chen, ASME J. Heat Transfer 124, 320 (2002).

${ }^{28}$ N. W. Ashcroft and N. D. Mermin, Solid State Physics (Saunders College, Fort Worth, TX, 1976).

${ }^{29}$ X. Y. Wang, D. M. Riffe, Y.-S. Lee, and M. C. Downer, Phys. Rev. B 50, 8016 (1994).

${ }^{30}$ G. Grimvall, in Selected Topics in Solid State Physics, edited by E. Whohlfarth (North-Holland, New York, 1981). 
${ }^{31}$ W. L. McMillan, Phys. Rev. 167, 331 (1968).

${ }^{32}$ P. B. Allen, Phys. Rev. Lett. 59, 1460 (1987).

${ }^{33}$ P. E. Hopkins, ASME J. Heat Transfer (in press).

${ }^{34}$ R. M. Martin, Electronic Structure: Basic Theory and Methods (Cambridge University Press, New York, 2004).

${ }^{35}$ S. Scandolo, P. Giannozzi, C. Cavazzoni, A. Pasquarello, and S. Baroni, Z. Kristallogr. 220, 574 (2005).

${ }^{36}$ J. P. Perdew and A. Zunger, Phys. Rev. B 23, 5048 (1981).

${ }^{37} \mathrm{We}$ used the Au.pz-d-rrkjus.UPF pseudopotential from the http:// www.quantum-espresso.org distribution.

${ }^{38}$ H. J. Monkhorst and J. D. Pack, Phys. Rev. B 13, 5188 (1976).

${ }^{39}$ D. R. Lide, CRC Handbook for Chemistry and Physics, 89th ed. (Internet Version, CRC Press, Boca Raton, FL, 2008).

${ }^{40}$ A. Dal Corso, A. Pasquarello, and A. Baldereschi, Phys. Rev. B 56, R11369 (1997).

${ }^{41}$ P. E. Blochl, O. Jepsen, and O. K. Anderson, Phys. Rev. B 49, 16223 (1994).
${ }^{42}$ J. K. Chen, D. Y. Tzou, and J. E. Beraun, Int. J. Heat Mass Transfer 49, 307 (2006).

${ }^{43}$ W. G. Vincenti and C. H. Kruger, Introduction to Physical Gas Dynamics (Krieger, Malabar, FL, 2002).

${ }^{44}$ T. Q. Qiu and C. L. Tien, ASME J. Heat Transfer 115, 842 (1993).

${ }^{45}$ F. Incropera and D. P. DeWitt, Fundamentals of Heat and Mass Transfer, 4th ed. (Wiley, New York, 1996).

${ }^{46}$ A. N. Smith and P. M. Norris, Appl. Phys. Lett. 78, 1240 (2001).

${ }^{47}$ A. Majumdar, ASME J. Heat Transfer 115, 7 (1993).

${ }^{48}$ T. Q. Qiu and C. L. Tien, ASME J. Heat Transfer 115, 835 (1993).

${ }^{49}$ P. E. Hopkins, J. C. Duda, R. N. Salaway, J. L. Smoyer, and P. M. Norris, Nanoscale Microscale Thermophys. Eng. 12, 320 (2008).

${ }^{50}$ C. K. Sun, F. Vallee, L. Acioli, E. P. Ippen, and J. G. Fujimoto, Phys. Rev. B 50, 15337 (1994).

${ }^{51}$ P. E. Hopkins and P. M. Norris, ASME J. Heat Transfer 131, 043208 (2009).

${ }^{52}$ P. E. Hopkins and P. M. Norris, Appl. Surf. Sci. 253, 6289 (2007). 